\title{
Cardiomiopatia Hipertrófica Tratada por Técnica Intervencionista de Redução Septal
}

\author{
José A ntonio Marin-N eto, Moysés 0 . Lima Fo, A ndré Schmidt, Benedito C. Maciel, Rogério Tumelero \\ Ribeirão Preto, SP
}

\begin{abstract}
Mulher de 75 anos com cardiomiopatia hipertrófica obstrutiva, com dispnéia classe IV, refratária ao tratamento clínico, apresentava contra-indicações relativas para abordagens cirúrgica e de implante de marcapasso. Realizou-se procedimento intervencionista para injeção seletiva de álcool absoluto no $1^{\circ}$ ramo septal da artéria interventricular anterior. O infarto septal provocado acompanhou-se de liberação enzimática, elevação de STe bloqueio de ramo direito do feixe de His. Não houve complicações inesperadas, e o gradiente da via ejetiva de ventrículo esquerdo, de $66 \mathrm{mmHg}$, foi imediatamente abolido. Controle ecocardiográfico evidencia manutenção desse resultado, até o momento, dois meses após o procedimento, em correspondência a marcante alívio sintomático.
\end{abstract}

A 75 year-old woman with obstructive hypertrophic cardiomyopathy and class IV dyspnea refractory to medical management had relative contraindications for both pacemaker implantation and surgical approach. Percutaneous intervention was devised to perform the selective injection of absolute alcohol in the first septal branch of the left anterior descending coronary artery. This led to a limited septal infarction documented by ST elevation, enzymatic elevation and the appearance of complete right bundle branch block on the electrocardiogram. A baseline left ventricular outflow tract pressure gradient of $66 \mathrm{mmHg}$ was immediately abolished and no unexpected complications supervened. Currently, two months after the procedure, marked symptomatic relief is present and associated with the absence of obstructive outflow gradient, as documented by echocardiography.
O tratamento da cardiomiopatia hipertrófica é tradicionalmente baseado no emprego de fármacos dotados de propriedades adequadas para amenizar os sintomas causados pela disfunção diastólica típica da doença. Quando ocorre importante obstrução da via ejetiva do ventrículo esquerdo (VE), e os sintomas decorrentes não são controlados, farmacologicamente, há o recurso cirúrgico da miotomia e/ ou miectomia; nesses pacientes, outra opção, ainda em investigação, consiste no implante de marcapasso cardíaco. Recentemente, técnica intervencionista capaz de promover redução septal e aliviar o gradiente obstrutivo foi descrita em grupos restritos de pacientes.

O objetivo deste relato é descrever a aplicação inédita no país, deste método intervencionista, com sucesso, em paciente com limitações para aplicação de outras formas terapêuticas.

Hospital das Cínicas da Faculdade de Medicina de Ribeirão Preto - USP Correspondência: José Antonio Marin-Neto - Faculdade de Medicina de Ribeirão Preto-USP - Av. Bandeirantes, 3900 - 14048-900 - Ribeirão Preto, SP Recebido para publicação em 5/1/98

Aceito em 4/3/98

\section{Relato do Caso}

Paciente com 75 anos, feminina, leucoderma, há cerca de 20 anos, apresentava dor precordial anginosa atípica e hipertensão arterial sistêmica. De 1979 a 1986 teve vários episódios de fibrilação atrial (FA) aguda transitória, relacionada com dispnéia de esforço e dor precordial. Nesse período, sempre detectou-se sopro mitral regurgitativo em repouso. Em 1986, o eletrocardiograma (ECG) evidenciava sobrecarga ventricular esquerda e o estudo ecocardiográfico demonstrava hipertrofia concêntrica do VE com discreto predomínio no septo interventricular $(20 \mathrm{~mm}$ contra $18 \mathrm{~mm}$ na parede posterior). Observou-se movimento sistólico anteriorizado do folheto anterior da valva mitral mas sem evidência de obstrução ao fluxo na via ejetiva do VE. Em 1989, pela primeira vez, quantificou-se gradiente intraventricular de $34 \mathrm{mmHg}$ ao ecocardiograma e acentuava-se a hipertrofia septal assimétrica. Isso coincidia com agravamento sintomático mas o estudo cineangiocardiográfico realizado na ocasião não evidenciou coronariopatia obstrutiva associada.

Desde 1979, a paciente foi medicada sucessivamente com betabloqueadores, bloqueadores de canais de cálcio, 


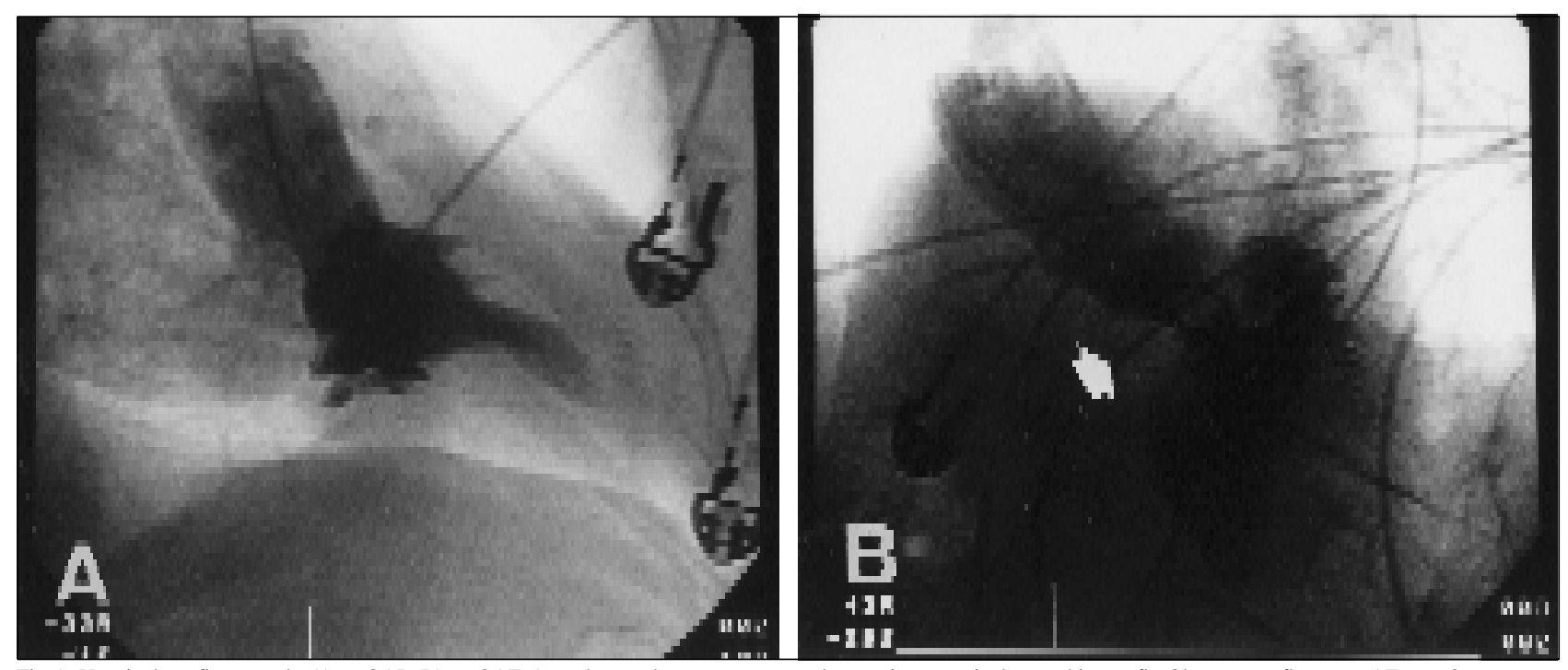

Fig. 1 - Ventriculografia esquerda: A) em OAD; B) em OAE. A seta branca demonstra a porção do septo interventricular com hipertrofia. Observar o refluxo para AE, grau 2.

inibidores da enzima de conversão, diuréticos e agentes antiarrítmicos para controle da FA paroxística (inclusive disopiramida).

Antecedentes significativos incluem pancreatite crônica agudizada em quatro episódios, de 1985 a 1990, coledocolitíase em 1984 e 1993, com tratamento por instalação de endoprótese no canal de Wirsung em 1993. Pai, mãe e irmão tiveram óbito de causa cardíaca, precocemente.

Em maio/97 foi internada com congestão pulmonar associada a novo episódio de FA aguda, e intensificação do gradiente intraventricular $-77 \mathrm{mmHg}$ ao ecocardiograma. Após quatro meses, novo episódio de congestão pulmonar nas mesmas condições de FA, que não pôde ser revertido a ritmo sinusal. Decidiu-se, então pela indução do infarto agudo do miocárdio por alcoolização de artéria septal. Este procedimento foi realizado com a paciente sendo tratada com verapamil $240 \mathrm{mg} / \mathrm{dia}$, amiodarona $200 \mathrm{mg} / \mathrm{dia}$ para controle cronotrópico em vigência de FA renitente e anticoagulação oral, em 15/10/97.

Efetuou-se estudo hemodinâmico direito/esquerdo, ventriculografia esquerda (fig. 1), e coronariografia pela técnica de Judkins. Constatou-se ausência de lesões coronárias obstrutivas significantes (fig. 2A), gradiente na via ejetiva ventricular esquerda pico-a-pico médio de $70 \mathrm{mmHg}$ (fig. 3A), e insuficiência mitral de grau moderado. Verificando-se existir $1^{\circ}$ ramo septal de bom calibre e extensão, optou-se por dar sequiência ao tratamento proposto. Pela artéria femoral esquerda introduziu-se bainha valvulada $6 \mathrm{~F}$ para a passagem de cateter pigtail até o VE, possibilitando se medir o gradiente interventricular durante o procedimento em relação ao cateter-guia JL 8F posicionado em coronária esquerda. Pela veia femoral direita instalou-se marcapasso provisório no ventrículo direito(VD). Monitorizou-se o traçado eletrocardiográfico, continuamente, em doze derivações.

Para cateterização seletiva do ramo septal da coronária interventricular anterior usou-se cateter-balão over thewire 2,0 × 20mm, avançado sobre corda-guia 0,014", após administração endovenosa de 15.000UI. de heparina, 40mg de meperidina, $0,2 \mathrm{mg}$ de morfina e $100 \mathrm{mg}$ de lidocaína (seguida de infusão de $1 \mathrm{mg} / \mathrm{min}$ durante todo o restante do procedimento). O cateter-balão foi insuflado em 6 atm (valor nominal $=2 \mathrm{~mm}$ ), em posição proximal do ramo septal (fig. 2B). O gradiente subaórtico diminuiu imediatamente de 66 para $5 \mathrm{mmHg}$. Retirou-se em seguida a corda-guia. Após se certificar de que, injetando-se contraste pela luz do cateterbalão, não havia refluxo para a artéria interventricular anterior, $4 \mathrm{ml}$ de álcool absoluto foi instilado por essa mesma via, durante $2 \mathrm{~min}$, distalmente no ramo septal ocluído pelo balão (fig. 2C).

Durante a alcoolização septal a paciente referiu dor precordial de discreta intensidade e se registrou imediato bloqueio de ramo direito (BRD), acompanhado de supradesnivelamento de ST de 0, $1 \mathrm{mV}$ em V1 e V2 (fig. 4A e 4B). Não ocorreu alteração hemodinâmica nem arritmias, e houve remissão espontânea da dor após alguns minutos. Coronariografia esquerda evidenciou artéria septal ocluída (TIMI0) (fig. 2D) e manometria mostrando desaparecimento total do gradiente intraventricular esquerdo (fig. 3B).

A paciente foi observada em unidade coronária por 72h, documentando-se pico de elevação da CKMB de 211 $\mathrm{U} / 1$ (VN até $25 \mathrm{U} / \mathrm{l})$. No $6^{\circ}$ dia após a embolização, o ecocardiograma confirmou a ausência de gradiente intraventricular esquerdo e evidenciou acinesia das regiões ínfero e ântero-basal do septo interventricular. Recebeu alta hospitalar nessa data, em uso de verapamil $240 \mathrm{mg} / \mathrm{dia}$, amiodarona $200 \mathrm{mg} /$ dia e anticoagulante oral.

No retorno de duas semanas após o procedimento, a paciente referia melhora importante dos sintomas, não mais apresentando dor precordial ou dispnéia aos pequenos e moderados esforços. ECG evidenciava reversão a ritmo sinusal e manutenção do padrão de BRD.

Dois meses depois da redução septal mantinha-se o alívio sintomático e não se registravam intercorrências ou alterações laboratoriais. 

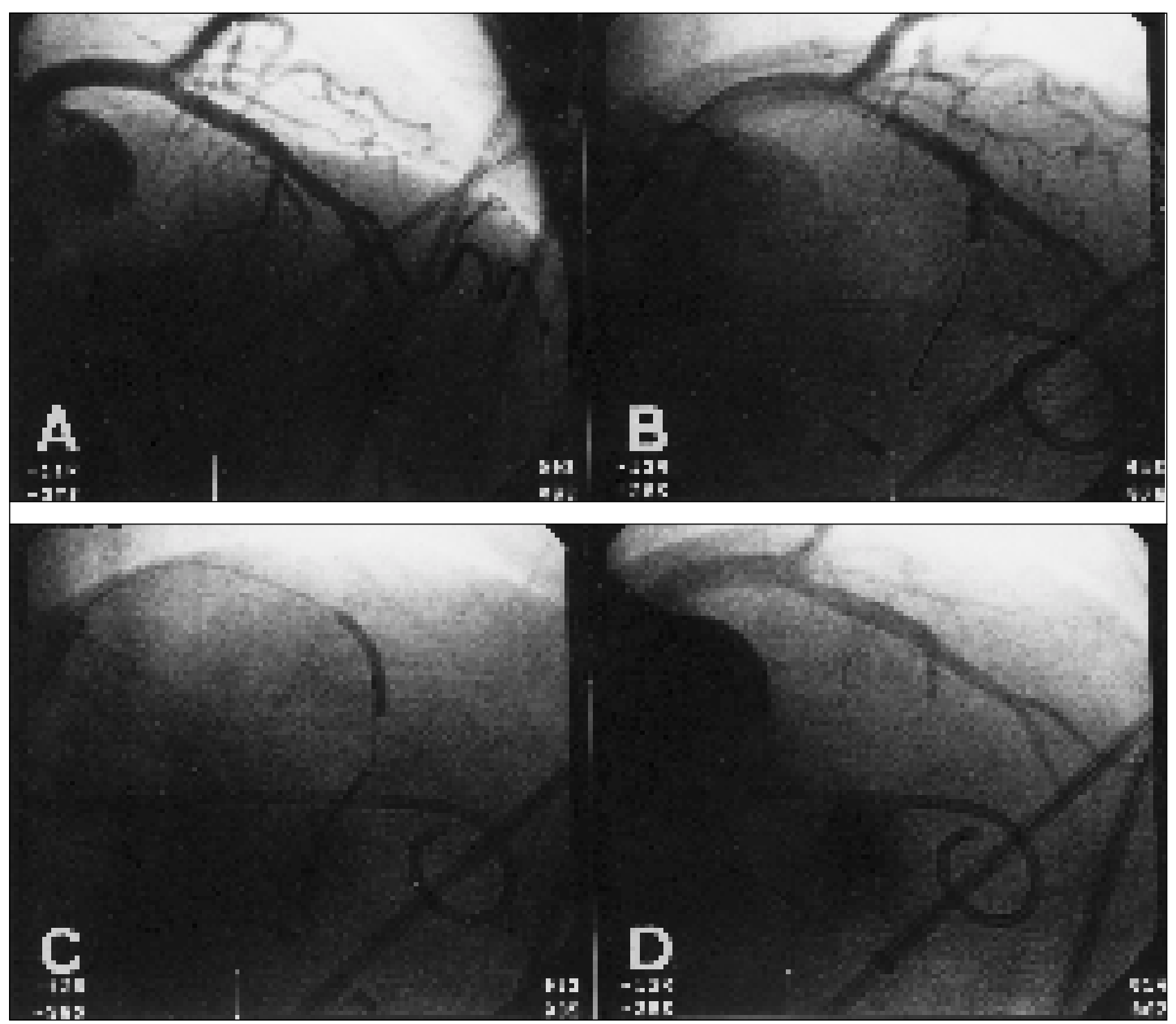

Fig. 2 - Coronária esquerda em PA cranial: A) antes da alcoolização septal; B) balão insuflado em artéria septal, fluxo livre em artéria interventricular anterior; C) momento da alcoolização da artéria septal; D) término do procedimento (artéria septal ocluída). Observar o cateter pigtail posicionado no ventrículo esquerdo durante o procedimento para a medida do gradiente pressórico.

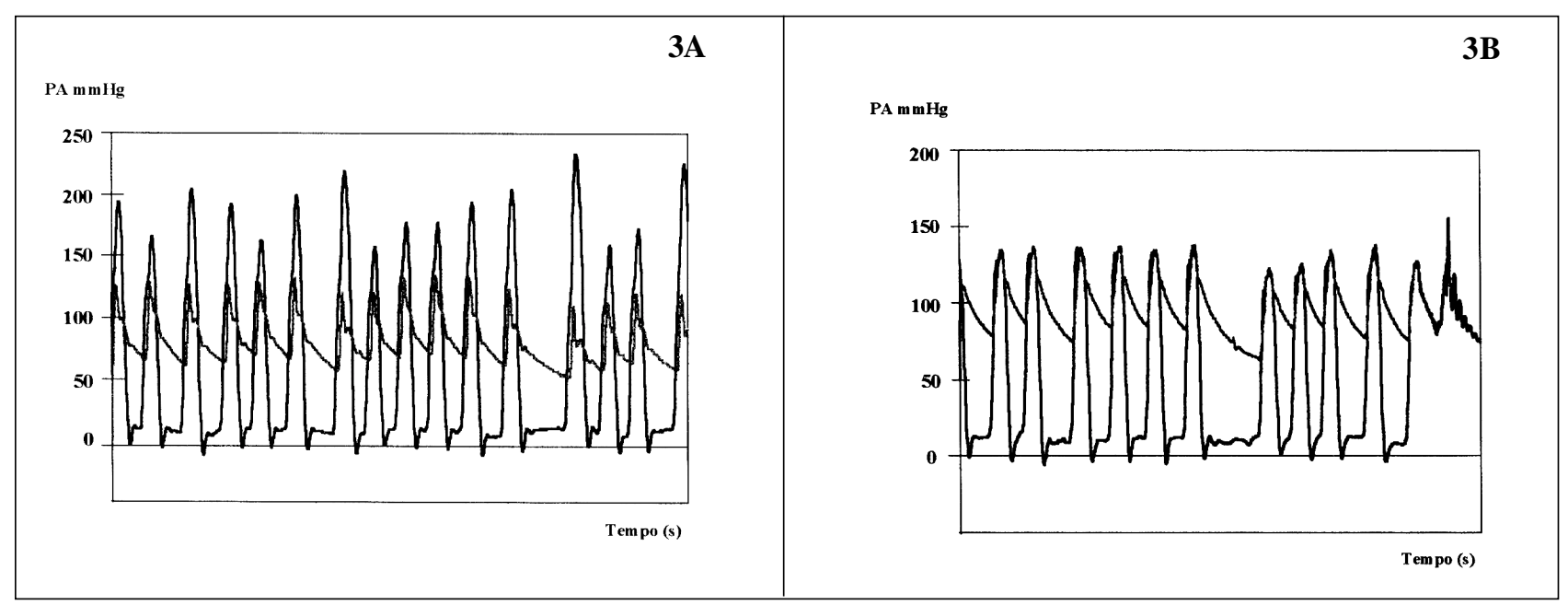

Fig. 3 - Registro simultâneo de pressões documenta gradiente sistólico entre a cavidade ventricular esquerda (em preto) e aorta (azul), em condição de repouso, antes do procedimento (painel 3a), e sua abolição após alcoolização septal (painel 3b). Observar o ritmo de fibrilação atrial. 


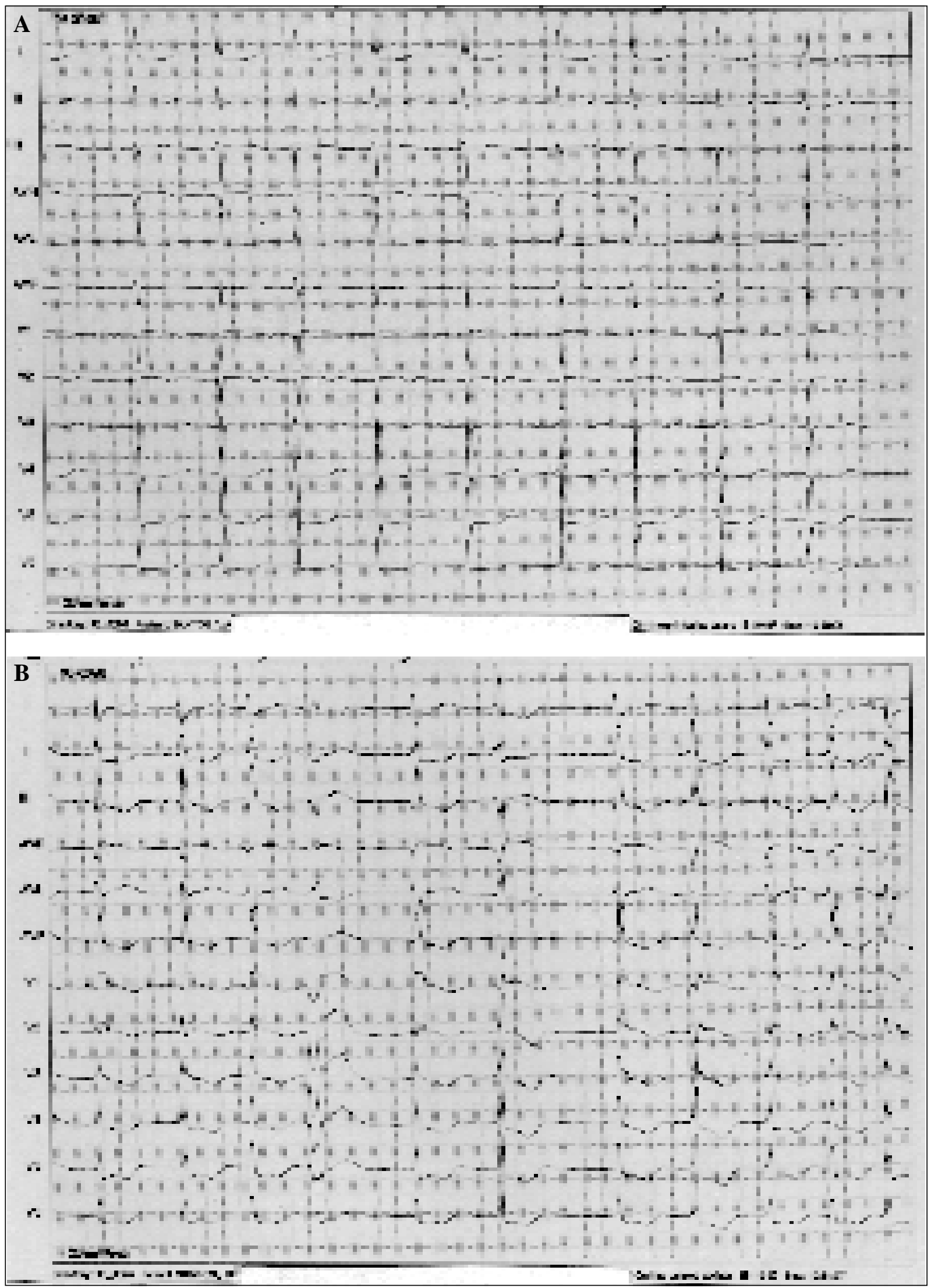

Fig. 4 - Eletrocardiograma de superfície em 12 derivações: A) imediatamente antes da alcoolização, às 10:51:08h; B) imediatamente após o inicio da injeção de álcool absoluto em artéria septal, às 10:51:37h. Observar a persistência do ritmo de fibrilação atrial, o aparecimento de extra-sistolia ventricular isolada, e de BRD, e a acentuação das alterações de repolarização ventricular (ST supra-desnivelado em V1-V2). 


\section{Discussão}

A cardiomiopatia hipertrófica, entidade mórbida relativamente prevalente, teve reconhecimento clínico há exatos 40 anos, a partir de relato original por Brock ${ }^{1}$. Nessa época, não se dispunham de métodos, como a ecocardiografia, e a própria angiocardiografia de contraste radiológico apresentava muito limitada resolução e aplicabilidade. Assim explica-se que a nova doença tenha sido inicialmente confundida, clinicamente, com a estenose valvar aórtica, inclusive pela indicação de correção cirúrgica, nessa experiência pioneira ${ }^{1}$. Também é compreensível que o distúrbio fisiopatológico responsável pelo mimetismo parcial da estenose aórtica valvar na cardiomiopatia hipertrófica, a ocorrência de obstrução dinâmica da via ejetiva do VE, tenha se constituído no mais fascinante aspecto da nova doença ${ }^{2}$.

É bem estabelecido hoje o conceito de que somente em minoria, ainda que expressiva, de doentes com cardiomiopatia hipertrófica, ocorra a variante obstrutiva sistólica ${ }^{3}$. Mas é justamente nesses casos que a doença tem manifestações mais ominosas e de mais difícil controle ${ }^{2,3}$. De fato, é nessas circunstâncias que, além do indefectível prejuízo de relaxamento e conseqüente redução da complacência ventricular, caracterizando obstrução diastólica, há obstáculo dinâmico à ejeção cardíaca. É comum, então, manifestar-se dispnéia, dor precordial, palpitações, e síncope de esforço. Morte súbita tem incidência baixa, variável de acordo com a população de doentes descrita ${ }^{2,3}$.

O manejo clínico habitual nessas circunstâncias apóia-se nos princípios de se evitar esforços competitivos, profilaxia de endocardite infecciosa, e administração de fármacos capazes de influenciar beneficamente as propriedades cronotrópicas e lusitrópicas ventriculares. Essas intervenções farmacológicas visam também neutralizar aumentos do inotropismo cardíaco provocados por estimulação simpática, e evitar a indução de arritmias cardíacas. Entre esses agentes farmacológicos, os mais indicados são os bloqueadores beta-adrenérgicos, os antagonistas dos canais de cálcio não taquicardizantes, e antiarrítmicos como a disopiramida e a amiodarona ${ }^{2-5}$.

Diversas intervenções cirúrgicas são classicamente empregadas em pacientes refratários ao tratamento clínico, quando o gradiente se torna $>50 \mathrm{mmHg}$ em repouso ${ }^{6-8}$, assim, a miotomia e miectomia septais, com ou sem concomitante substituição valvar mitral ${ }^{9,10}$. Este último método cirúrgico, usualmente mandatório para insuficiência mitral grave, também pode ser utilizado com a intenção de abolir a participação da válvula na criação do gradiente obstrutivo intraventricular ${ }^{11}$. Mais recentemente, a abordagem cirúrgi- ca desta condição mórbida enriqueceu-se com a proposição de outra técnica, mais conservadora, a de se associar à miectomia septal a extensão longitudinal do folheto anterior mitral com enxerto de pericárdio ${ }^{12}$.

Embora ainda não haja completo entendimento de seu mecanismo, e seus efeitos permaneçam em fase de investigação, o implante de marcapasso bicameral constitui método promissor em pacientes com a forma obstrutiva da cardiomiopatia hipertrófica ${ }^{13-16}$. Aparentemente, reduz-se o gradiente sistólico em proporção ao alívio sintomático, e também se produz melhora das propriedades diastólicas, inclusive por certo grau de involução da hipertrofia ven$\operatorname{tricular}^{15,16}$.

Mais recentemente, foram relatados resultados preliminares da aplicação de técnica intervencionista percutânea, para produção de infarto septal e, conseqüente, redução do fenômeno obstrutivo sistólico na cardiomiopatia hipertrófica ${ }^{17,18}$. Esses relatos, pertinentes a 18 pacientes estudados na Europa, foram reproduzidos em centro norte-americano, em outro grupo de 18 casos similares ${ }^{19}$.

No presente relato foram confirmadas as expectativas geradas com esses dois estudos. A paciente em tela constitui exemplo de evolução clínica prolongada da cardiomiopatia hipertrófica, com agravamento progressivo dos sintomas para forma intratável clinicamente, a despeito do uso de todo o arsenal farmacológico, em fases distintas e sucessivas. O implante de marcapasso cardíaco foi contraindicado pela freqüente reincidência de surtos de FA, com difícil controle farmacológico e elétrico. A idade avançada, seu estado geral combalido pela presença de co-morbidades e sua resistência psicológica a aceitar a proposição de tratamento cirúrgico, induziram à tentativa do procedimento percutâneo.

O método de redução septal, aqui em pauta, tem seu mecanismo de ação dependente do infarto produzido pelo efeito tóxico direto do álcool sobre o miocárdio, associado à oclusão total da artéria responsável pela nutrição dessa área, que imediatamente sofre intenso vasoespasmo, como predito experimentalmente ${ }^{20}$.

Pode-se concluir do relato desta experiência inicial, que o método de redução septal intervencionista é reprodutivelmente efetivo para abolir a obstrução sistólica na cardiomiopatia hipertrófica. Sob o aspecto técnico, é relativamente simples, por sua aplicabilidade derivada de outros procedimentos percutâneos. Seu real papel no tratamento de pacientes com a doença permanece a ser estabelecido. Neste sentido, porém, sua situação não é diversa das outras opções terapêuticas disponíveis, para as quais ainda inexiste comprovação de efeitos favoráveis sobre a história natural da cardiomiopatia hipertrófica.

\section{Referências}

1. Goodwin JF - Congestive and hypertrophic cardiomyopathies. A decade of study. Lancet 1970; I: 731-3.

2. McKenna W, Dearfield J, Feruqui A et al - Prognosis in hypertrophic cardiomy- opathy: role of age, clinical, electrocardiographic and hemodynamics features. Am J Cardiol 1981; 47: 532-8.

3. Maron BJ, Bonow RO, Cannon RO et al - Hypertrophic cardiomyopathy: inter- 
relations of clinical manifestations, pathophysiology, and therapy. N Engl J Med 1987; 316: 780-9.

4. McKenna W, Oakley CM, Kirkler DM et al-Improved survival with amiodarone in patients with hypertrophic cardioyopathy and ventricular tachycardia. $\mathrm{Br}$ Heart J 1985; 53: 412-16.

5. Sherrid M, Delia E, Dwyer E-Oral disopyramide therapy for obstructive hypertrophic cardiomyopathy. Am J Cardiol 1988; 62: 1085-8.

6. Morrow AG, Reitz BA, Epstein SE et al - Operative treatment in hypertrophic subaortic stenosis: techniques, and the results of pre and post-operative assessment in 83 patients. Circulation 1975; 52: 88-102.

7. Borer JS, BacharachSL, Green MV etal-Effect of septal myotomy and myectomy on left ventricular systolic function at rest and during exercise in patients with IHSS. Circulation 1979; 60(suppl I): I-82-I-7.

8. Maron BJ, Epstein SE, Morrow AB et al - Symptomatic status and prognosis of patients after operation for hypertrophic obstructive cardiomyopathy: efficacy of ventricular septal myotomy and myectomy. Eur Heart J 1983; 4(suppl F): 17585.

9. Leachman RD, KrajcerZ, Azic R, Cooley DA - Mitral valve replacement in hypertrophic cardiomyopathy: ten year follow-up in 54 patients. Am J Cardiol 1987; 60: 1416-18.

10. Krajcer Z, Leachman RD, Cooley DA et al - Mitral valve replacement and septal myomectomy in hypertrophic cardiomyopathy: ten year follow-up in 80 patients. Circulation 1988; 78: 135-43.

11. Schulte HD, Bircks W, Lösse B, Godehardt E, Schwartzkopff B - Prognosis of patients with hypertrophic obstructive cardiomyopathy after transaortic myectomy. Late results up to twenty-five years. J Thorac Cardiovasc Surg 1993; 106:
709-17.

12. Kofflard MJ, Van Herwerden LA, Waldstein DJ et al - Initial results of combined anterior mitral leaflet extension and myectomy in patients with obstructive hypertrophic cardiomyopathy. J Am Coll Cardiol 1996; 28: 197-202.

13. Fananapazir L, Canon III RO, Tipodi D et al - Impact of dual chamber permanent pacing in patients with obstructive hypertrophic cardiomyopathy with symptoms refractory to verapamil and Beta-adrenergic blocker therapy. Circulation 1992; 85: 2149-61.

14. Jeanrenaud X, Goy JJ, Kappenberger L-Effects of dual-chamberpacing in hypertrophic obstructive cardiomyopathy. Lancet 1992; 339: 1318-23.

15. Fananapazir L, Epstein MD, Curiel RV et al - Long-term results of dual-chamber pacing in obstructive hypertrophic cardiomyopathy: evidence for progressive symptomatic and hemodynamic improvement and reduction of left ventricular hypertrophy. Circulation 1994; 90: 2731-42.

16. Nishimura RA, Hayes DL, Ilstrup DM, Holmes DR, Tajik AJ - Effect of dual chamber pacing on systolic and diastolic function in patients with hypertrophic cardiomyopathy. J Am Coll Cardiol 1996; 27: 421-30.

17. Sigwart U - Non-surgical myocardial reduction for hypertrophic obstructive cardiomyopathy. Lancet 1995; 346: 211-4.

18. Knight C, Kurbaan AS, Seggewiss H et al - Nonsurgical septal reduction hypertrophic obstructive cardiomyopathy. Outcome in the first series of patients. Circulation 1997; 95: 2075-81.

19. Spencer III WH - Nonsurgical septal reduction in hypertrophic obstructive cardiomyopathy. ACC Educational Highlights/Fall 1997; 12: 1-4.

20. Rogers PJ, Bove AA - Epicardial coronary artery constriction with intravenous ethanol. Int J Cardiol 1989; 29: 301-10. 